SCRDSI-2021, Aravali Institute of Technical Studies, Udaipur (Rajasthan), India

International Journal of Technical Research \& Science (Special Issue) ISSN No.:2454-2024 (online)

\title{
WIRELESS SOLAR POWER TRANSMISSION
}

\author{
Lalit Kumar prajapat, Narendra Kumar Yadav \\ E-Mail Id: lalitkprajapat98@gmail.com,_skynarendra73@gmail.com \\ Department of Electrical Engineering, Aravali Institute of Technical Studies, Udaipur, India
}

\begin{abstract}
The futuristic advances in power transmission through microwaves. Sun is a limitless source of energy. A space power satellite (sps) orbiting round the earth traps solar energy \& generates electric power using photovoltaic cells of sizable area. Sps transmits the generated power via a microwave beam to the receiving rectenna site on earth. A rectenna (rectifying antenna) comprises of a mesh of dipoles \& diodes for absorbing microwave energy from a transmitter \& converts it into electric power. We are aware of the fact that light is an electromagnetic wave. Light rays never diffuse in space \& if by any means these rays can be transmitted from space to earth then it will be a perfect solution for our desired need of $24 \mathrm{hrs}$ power supplies. The approaches for establishing human race in space can come true only if the basic requirement of human beings is satisfied i.e. 24HRS power, which can be efficiently served by rectenna. A wireless power transmission using microwave is a system which contains satellite based solar power system (SPS). The use of solar cells in space achieves 24 hour sunlight $\&$ are unaffected by atmosphere \& clouds. My concept is a comprehensive study of various components of satellite based SPS \& projects this technology as a bulk source of power generation in future.
\end{abstract}

Keywords: Wireless power transfer, microwave power transmission, solar power satellite

\section{INTRODUCTION}

The present electricity generation system is not very efficient in terms of energy transfer. About 20 to $30 \%$ energy is lost during the distribution of the electricity. Therefore the scientists are working on the projects to improve the ultimate electricity supply. Scientists are looking for alternate \& efficient technologies to provide $100 \%$ electricity transfer. The change \& development in the various fields have brought more client satisfaction $\&$ output. Therefore the wireless transmission of electricity is also on move. A major problem

Facing Planet Earth is provision of an adequate supply of clean energy. It has been that we face "...three simultaneous challenges population growth, resource consumption, \& environmental degradation all converging particularly in the matter of sustainable energy supply." It is widely agreed that our current energy practices will not provide for all the world's peoples in an adequate way still leave our Earth with a livable environment. Hence, a major task for the new century will be to develop sustainable environmentally friendly sources of energy.

Projections of future energy needs over this new century show an increase by a factor of at least two \& one Half, perhaps by as much as a factor of five. All of the scenarios from references indicate continuing use of fossil sources nuclear, \& large hydro... It will be a Herculean task to acquire this projected amount of energy. Thus, unlike systems for the terrestrial capture of solar, a space-based system would not be limited by the vagaries of the day-night Cycle. Furthermore, if the transmission frequency is properly chosen, delivery of power can be carried out essentially independent of weather conditions. Thus Space Solar Power could provide base load electricity.

\section{WIRELESS ELECTRICITY TRASMISSION (WET) TECHNOLOGY}

Wireless power transmission is a process that takes place in any type of system in which electrical current is conveyed from a power source to an electrical load. What makes this process unique is that there is no usage of any type of wiring to connect the system to a source of power. Wireless electricity (Power) transmission basically is the transmission of electricity with the help of microwaves \& there is no need to use cables, towers $\&$ grid stations .

\section{MICROWAVE ENERGY TRASNFER}

It is possible to achieve a long range using this method. In this method, microwave is sent to the long distances which are received through rectenna. Rectenna extracts microwave energy back to electrical energy. The main problem with this particular strategy is how the diameter of antenna needs to be order of kilometer. Power transmission via radio waves can be produced more directional, allowing longer distance power beaming, with shorter wavelengths of electromagnetic radiation, typically in the microwave range. Rectenna conversion efficiencies exceeding $95 \%$ are actually realized. 


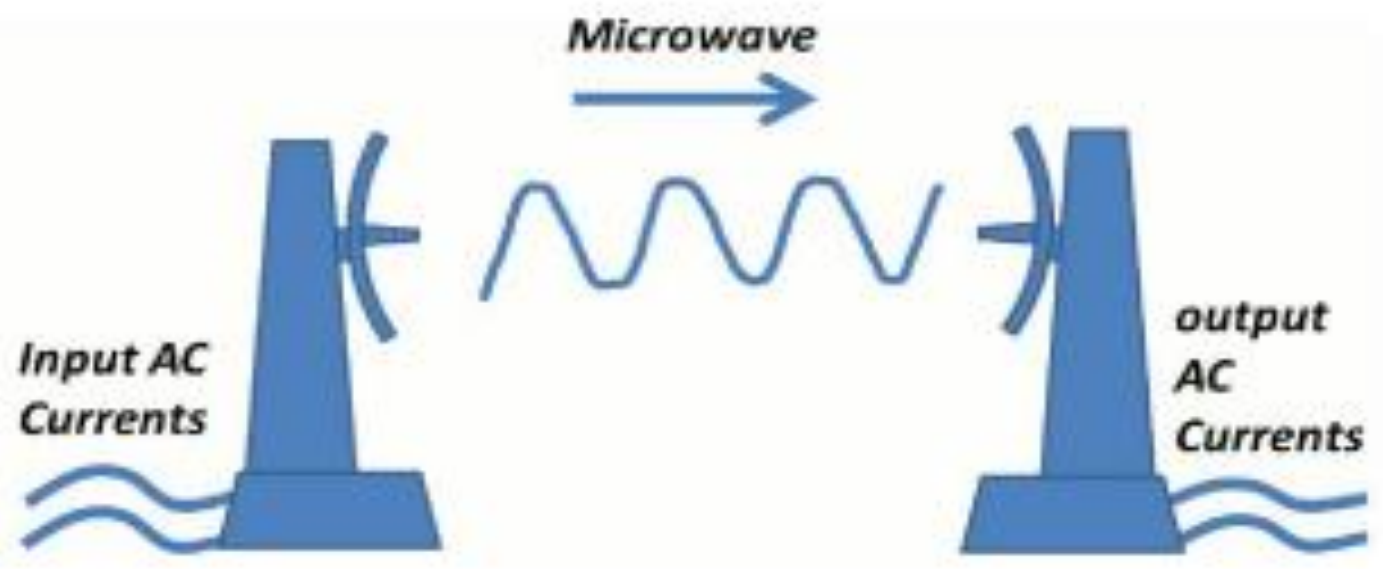

Fig. 3.1 Radio/Microwave Energy Transfer

\section{LASER BEAM TRASNFER}

This particular method, laser is beamed for the photovoltaic cells which extract the electrical energy. This method is quite challenging to implement and manage. This technology is slightly different with MPT where it enables the power concentrated in a small area by utilizing the mirror. This technology also produces high powers that are coherent and not dispersed. However, laser technology gets attenuated when it propagates through atmosphere. In addition, this technology has been used to apply to a rover to explore the presence of ice in the bottom of craters of the moon where no sunlight is available. On the other hand, the solar energy generated by the radiation is converted into the electric energy. This energy next will be converted to the laser light.

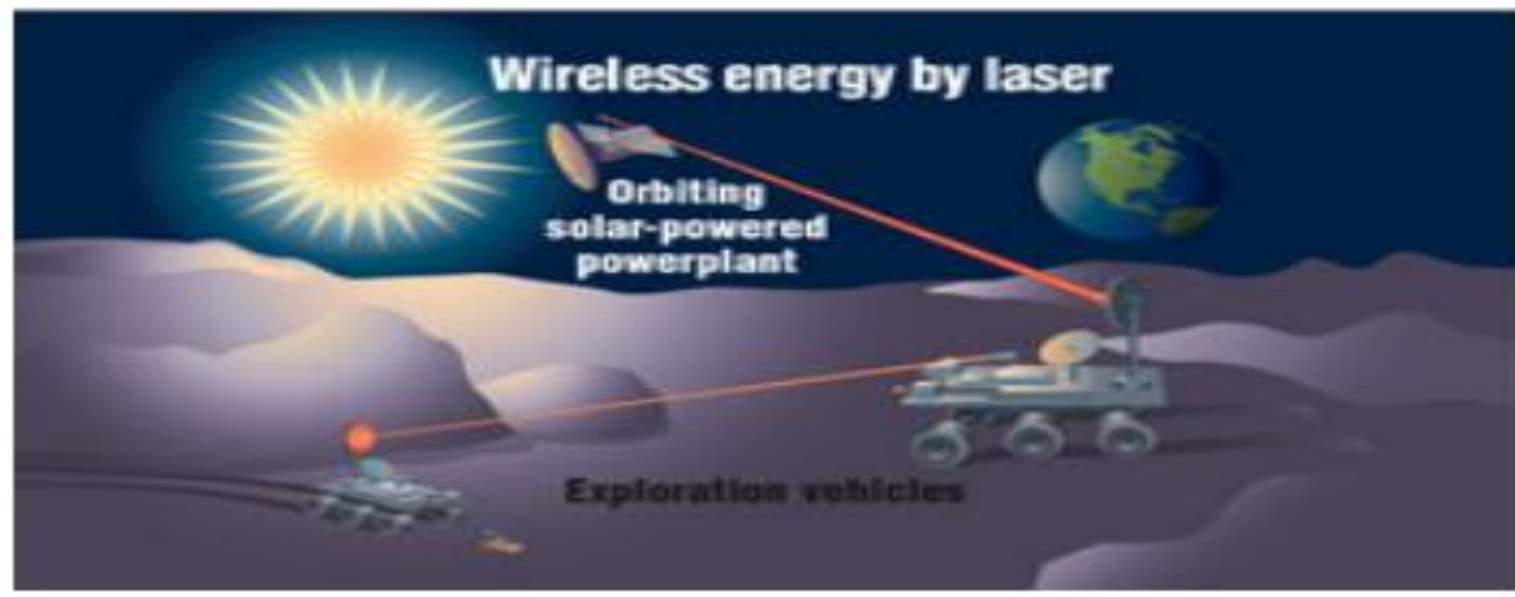

Fig. 4.1 Radio/Microwave Energy Transfer

\section{RECENT TECHNOLOGIES}

Utilization of resonance has rapidly grown in recent years to enhance the efficiency of wireless energy transfer in a wide variety of applications. In addition, the necessary core components of electronic product are being developed by electronic companies to help speed the introduction of the technology into niche applications. This will boost our creative capacities to bring much more substantial changes in technology so that can be implemented in particular tasks. Some of these revolutionary applications have been launched into market, while others are not ready yet for the commercial market. For instance, automotive charging is a novel innovation but not available into market because of needing standardization in its charging infrastructure. On the contrary, a breakthrough innovation for traditional inductive charging in mobile electronic has already developed by a consortium company. To ensure that multi-vendor products can charge anywhere in a common wireless ecosystem, the Standards Development Organizations (SDOs) is working to construct the interoperability standards in mobile devices for highly resonant wireless power transfer. All these efforts are paving the way toward new trend of wireless power technology, in which can be deployed in many applications.

\section{QI TECHNOLOGY}

This technology uses the small inductors to transmit power over higher frequencies and also support a charging distance of a few centimeters at most. As a result, portable devices have to be placed quite specifically on the 
SCRDSI-2021, Aravali Institute of Technical Studies, Udaipur (Rajasthan), India

International Journal of Technical Research \& Science (Special Issue) ISSN No.:2454-2024 (online)

dock for avoiding the shortage of a large magnetic field. Owing to its limitation on charging area, Qi components can use multiple resonator arrays to create a larger charging area. However, it still does not mitigate the problem and even wasting a lot of power to have individual coils switched on. In order to keep a strong enough connection, users therefore need to align their devices precisely with the magnetic field.

\section{ALLIANCE FOR WIRELESS POWER (A4WP) TECHNOLOGY}

A4WP is a next-generation of wireless power transfer enabling the efficient transfer of power to electronic devices. This is based on reference power transmitting and receiving resonators without the use of interconnecting wires. This technology allows multiple devices to be charged with differing power requirements from a single transmitter at any one time. Because this technology uses a larger electromagnetic field rather than the small inductor coils, therefore it enables devices to be charged without having to line-up precisely with the coil. Although A4WP has not released to the market yet the existence of this technology enables the electronic devices to be charged in any positions including Z-axis.

\section{SOLAR POWER SATELLITE}

There are several advantages to SPS. Solar radiation can be more efficiently collected in space, where it is roughly three times stronger than on the surface of the Earth \& it can be collected 24 hours per day (since there are no clouds or night in high Earth orbit). SPS does not use up valuable surface area on the Earth $\&$ can be beamed to areas with the highest dem\& at any particular time. Most of these systems would utilize photovoltaic (PV) cells similar to those on Earth-based systems (such as those used by home power systems \& highway sign panels). Others would utilize reflectors \& mechanical collectors similar to those used in special large-scale solar facilities in France \& the California desert (Barstow).

Most of these systems collect solar energy in space \& transmit it via a microwave energy beam to an Earthbased rectenna which converts the beam into electricity for use on Earth. In fact, telephone companies have been beaming microwaves through the atmosphere for over thirty years without any known problems.

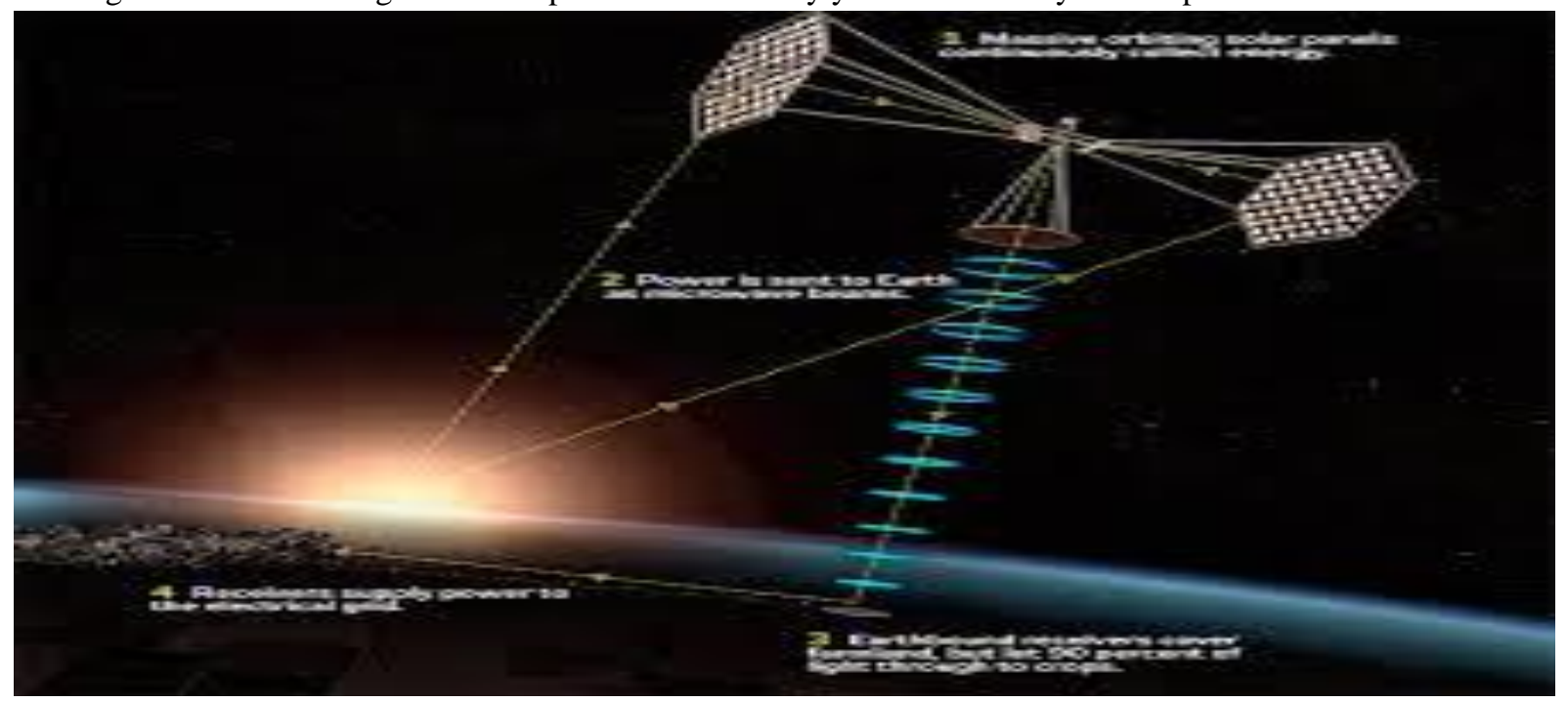

Fig. 8.1 Solar Power Satellite

\section{ADVANTAGE AND DISAADVANTAGE}

\subsection{Some of the Advantages are as Follows}

$>$ Various ways of transmitting power wirelessly have been famous for centuries. The most widely known example is non-particulate radiation, for example radio waves. While such radiation is extremely good for wireless transmission of knowledge, it's not at all feasible to apply it for power transmission. Since radiation spreads in all directions, a massive tastes power would become wasted into free space.

$>$ Wireless Power Transmission system would completely eliminates the previous high-tension power transmission line cables, towers and sub stations involving the generating station and consumers and facilitates the interconnection of electrical generation plants with a global scale.

$>$ It's more freedom of both receiver and transmitters. Even mobile transmitters and receivers might be chosen to the WPT system.

$>$ The power could possibly be transmitted towards places the location where the wired transmission isn't feasible. Decrease of transmission is negligible level from the Wireless Power Transmission; therefore, the efficiency with this way is a lot higher than the wired transmission.

DOI Number: https://doi.org/10.30780/specialissue-SCRDSI-2021/010

pg. 39 
SCRDSI-2021, Aravali Institute of Technical Studies, Udaipur (Rajasthan), India

International Journal of Technical Research \& Science (Special Issue) ISSN No.:2454-2024 (online)

$>$ Power can be purchased with the rectenna provided that the WPT is operating. The power failure because of short and fault on cables could not exist from the transmission and power theft will be not possible in any respect.

\subsection{Some of the disadvantages are as follows}

$>$ High capital cost for practical implementation of wireless power transmission.

$>$ Another potential disadvantage is the interference of the microwaves with the present wireless communication system.

$>$ The effect of microwave radiations at high doses received is not suitable to human health.

\section{APPLICATION OF WIRELESS POWER TRASNMISSION}

$>$ 1. Moving targets for example fuel free airplanes, fuel free electric vehicles, moving robots and fuel free rockets. Another applying WPT are wireless power source, wireless sensors and RF power adaptive rectifying circuits (PARC).

$>$ 2. Mobility - user device might be moved easily in the wireless range.

$>$ 3. Neat and easy Installation - since no cable running occasionally, just start-up the wireless device and you're ready to rumble.

> 4. Generating power by placing satellites with giant solar arrays in Geosynchronous Earth Orbit and transmitting the power as microwaves on the earth called Solar Power Satellites (SPS) will be the largest application of WPT

\section{CONCLUSIONS}

The concept of wireless power transmission is presented. There recent technological applications that make the human life more beneficial in the present world have been discussed.

Three new standard of wireless power technology that is already in competition with each other is also one of the talks of the topic in near future when other more standards are coming soon. Among these three wirelesses charging standards, which are going to be win in the race that will be defined by their recent great applications. From the comparison table it shows that A4WP standards which has the huge magnetic field and large charging distance must be keep ahead this technology then other standards whereas Qi and PMA also improving very fast.

More applications that are in under research with wireless power charging and in the field of robotics will be in our daily uses only if wireless power keeps improving.

\section{REFERENCES}

[1] Nikola Tesla 1905 Electrical World \& Engineer the Transmission of Electrical Energy Without Wires as a Means for Furthering Peace, p 21.

[2] Gernsback, Hugo 1919 Nikola Tesla \& His Achievements, Electrical Experimenter p 615.

[3] Nikola Tesla Nikola Tesla on His Work with Alternating Currents \& Their Application to Wireless Telegraphy, Telephony \& Transmission of Power $\mathrm{p} 126$.

[4] Dave Baarman\& Joshua Schwannecke 2009 Underst\&ing Wireless Power p 1.

[5] L, Geoffrey A 2006 IEEE 4th World Conference on Photovoltaic Energy Conversion 2.

[6] S.Sheik Mohammed, K. Ramasamy, T. Shanmuganantham, 2010, "Wireless Power Transmission - A Next Generation Power Transmission System" IEEE International Journal of Computer Applications. 